\title{
Chitin and Nanoquitin Preparation From Mush- room By-products Edible (Agaricus Bisporus) and River Crab (Procambarus Clarkii)
}

\section{Preparación de Quitina y Nanoquitina a Par- tir de Subproductos de Hongos Comestibles (Agaricus Bisporus) y Cangrejo de Río (Pro- cambarus Clarkii)}

I International Congress of Biotechnology, Environment, Chemistry and Food

Corresponding Author:

Alberto Renato Inca-Torres

creny_11@hotmail.com

Published: 29 August 2021

Production and Hosting by

Knowledge $E$

(c) Alberto Renato Inca-Torres et al. This article is distributed under the terms of the Creative Commons Attribution License, which permits unrestricted use and redistribution provided that the original author and source are credited.
Alberto Renato Inca-Torres ${ }^{1,2}$, Anabell Del Rocío Urbina-Salazar ${ }^{2,3}$, Valeria Fernanda Inca-Torres ${ }^{4}$, Bryan Anthony Urbina-Salazar ${ }^{1}$, and Juan Bautista ${ }^{3}$

${ }^{1}$ Universidad Técnica de Ambato, Facultad de Ciencias de la Salud, Carrera de Medicina, Ambato, Ecuador

${ }^{2}$ Universidad Técnica de Manabí, Instituto de Ciencias Básicas, Portoviejo, Ecuador

${ }^{3}$ Universidad de Sevilla, Facultad de Farmacia, Departamento de Bioquímica y Biología Molecular, Sevilla, España

${ }^{4}$ Hospital Básico Baños, Baños, Ecuador

\section{Abstract}

Chitin production processes involve highly polluting treatments, current studies have shown the use of biological methods gave better results because it preserves chitin structure. In this work, chitin was obtained from Agaricus bisporus and Procambarus clarkii following friendly environmental alternative procedures, through a sequential process based on the use of proteases, glucanases. Transformation of microfibrils into nanofibers was accomplished dissolving amorphous regions followed by acid disruption.

The chitin concentration determined as $\mathrm{N}$-acetyl glucosamine is $83 \pm 1.8 \%$ and $80 \pm 2.4 \%$ for $A$. bisporus and $P$. clarkii, respectively, being a good-quality chitin, similar to the commercially available one. Finally, highly uniform, approximately 8 nanometer-wide chitin nanofibers were obtained, which still maintained their original chemical and crystalline structures. The product can be used for industrial applications in pharmacy, cosmetics, agriculture, and wastewater treatment.

Keywords: Byproduct of mushroom, Fraction of chitin, Agaricus bisporus, , Procambarus clarkii

\section{Resumen}

Los procesos de producción de quitina implican tratamientos altamente contaminantes, en estudios actuales se ha demostrado que la utilización de métodos biológicos dieron mejores resultados porque preserva la estructura de la quitina. En este trabajo se obtuvo quitina tanto de Agaricus bisporus como de Procambarus clarkii siguiendo procedimientos alternativos amigables con el medio ambiente, mediante un proceso secuencial basado en el uso de proteasas, glucanasas. La transformación de microfibrillas en nanofibras se logró disolviendo las regiones amorfas seguido de la ruptura ácida. La concentración de quitina determinada como $\mathrm{N}$-acetil-glucosamina es de $83 \pm 1,8 \%$ y $80 \pm 2,4 \%$, para $A$. bisporus y $P$. clarkii respectivamente, siendo una quitina de buena calidad, similar a la 
disponible comercialmente. Finalmente se obtuvo nanofibras de quitina altamente uniformes con un ancho de aproximadamente $8 \mathrm{~nm}$ que aún mantenían sus estructuras químicas y cristalinas originales. El producto puede ser utilizado para aplicaciones industriales en farmacia, cosmética, agricultura y tratamientos de aguas residuales.

Palabras Clave: Subproducto de champiñón, Fracción de Quitina, Agaricus bisporus, Nanoquitina, Procambarus clarkii

\section{Introducción}

La pared de los hongos, el exoesqueleto e intestino de los insectos y exoesqueleto de crustáceos está constituido por un compuesto nanoestructurado basado principalmente en microfibrillas de quitina. La quitina es un polisacárido cristalino de alto peso molecular, esta estructura al hidratarse forma un compuesto duro, fuerte y resistente. El exoesqueleto de los crustáceos además de estar constituido por quitina, contiene partículas de carbonato de calcio y proteínas [1], y en el caso de los hongos están constituidos por quitina que se presenta en forma microfibrilar unida a glucanos, formando de esta manera un complejo fibrilar insoluble en álcali [2]. La quitina tiene propiedades únicas que permiten su uso en una amplia gama de aplicaciones en diferentes áreas (biotecnología, medicina, farmacia, agricultura, etc.). Enormes cantidades de residuos de champiñón y cangrejo de río son desechadas por las grandes industrias del procesamiento ocasionando contaminación al medio ambiente, es por ello que su reutilización sería una buena alternativa [3-6]. La quitina de los crustáceos es de uso frecuente, sin embargo, la presencia del mineral dificulta el proceso de extracción y lo métodos tradicionales para la preparación comercial de este tipo de quitina son altamente contaminantes [7].

La transformación de microfibrillas en nanocristales/nanofibras se puede lograr disolviendo las regiones amorfas seguido de una ruptura ácida, y debido a sus innumerables propiedades pueden ser utilizadas en la industria médico-farmacéutica y en la industria alimentaria para combatir la actividad antifúngica [8-11], debido a que es biocompatible, biodegradable y no es tóxica, etc. [12]. En este trabajo se describen procedimientos biotecnológicos amigables con el medio ambiente para la preparación de quitina y nanoquitina a partir de estos subproductos. Los productos obtenidos poseen numerosas aplicaciones prometedoras en diferentes industrias.

\section{Materiales y Métodos}

\subsection{Preparación de la materia prima}

Se utilizaron subproductos procedentes de la industria del champiñón (Agaricus bisporus) (TCH; Tallos de Champiñón) y subproductos procedentes de la industria del procesamiento del cangrejo de río (Procambarus clarkii) (C; Cangrejo), constituido 
fundamentalmente por el cefalotórax y el exoesqueleto de las colas, estos subproductos fueron secados, molidos y tamizados obteniendo un polvo fino al que se les denominó HTCH; Harina de Tallos de Champiñón y HC; Harina de Cangrejo, que se usó como material de partida, En el caso de los subproductos de cangrejo estos fueron previamente desmineralizados, mediante un método basado en la producción in situ de ácido/fermentación láctica [13].

\subsection{Preparación de la quitina}

Se utilizó un procedimiento secuencial mediante un tratamiento enzimático y posteriormente un tratamiento térmico. La desproteinización se llevó a cabo mediante un tratamiento enzimático con proteasas a pH 8,5 y $55^{\circ} \mathrm{C}$ con baja agitación (100 rpm) usando una relación $\mathrm{E} / \mathrm{S}$ de 0,01 . Las proteasas utilizadas han sido obtenidas mediante procesos biotecnológicos utilizando como materia prima lodos de depuradora crecidos con B. licheniformes [14].

\subsection{Caracterización de la quitina}

El porcentaje de quitina en base seca se determinó gravimétricamente después de la desproteinización [15], el contenido en hidratos de carbono totales se determinó mediante el método colorimétrico de Dubois, usando ácido sulfúrico-fenol [16] y el nitrógeno total $(\mathrm{Nt})$ se determinó mediante el método Kjeldahl de acuerdo con el procedimiento establecido por la A.O.A.C. 1995 [17], en un equipo automático KjeltecTM 840 (Foss Iberica S.A., Barcelona) equipado con digestor y destilador.

\subsection{Análisis infrarrojo}

Los espectros infrarrojos de las muestras se registraron usando pellets de bromuro de potasio con un espectrómetro FT-IR (FTIR 8300, Shimadzu).

\subsection{Preparación de nanofibras de quitina}

La preparación de nanofibras de quitina se llevó a cabo desintegrando los agregados de quitina mediante un proceso de molienda en una trituradora de tipo húmedo, generando suspensión de gel de quitina. La desintegración se logró debido a una alta relación superficie-volumen.

\section{Resultados}

Por lo general, los procesos de producción de quitina implican tratamientos altamente contaminantes, en este trabajo se obtuvo quitina tanto de $A$. bisporus como de $P$. clarkii mediante procedimientos alternativos amigables con el medio ambiente, para 


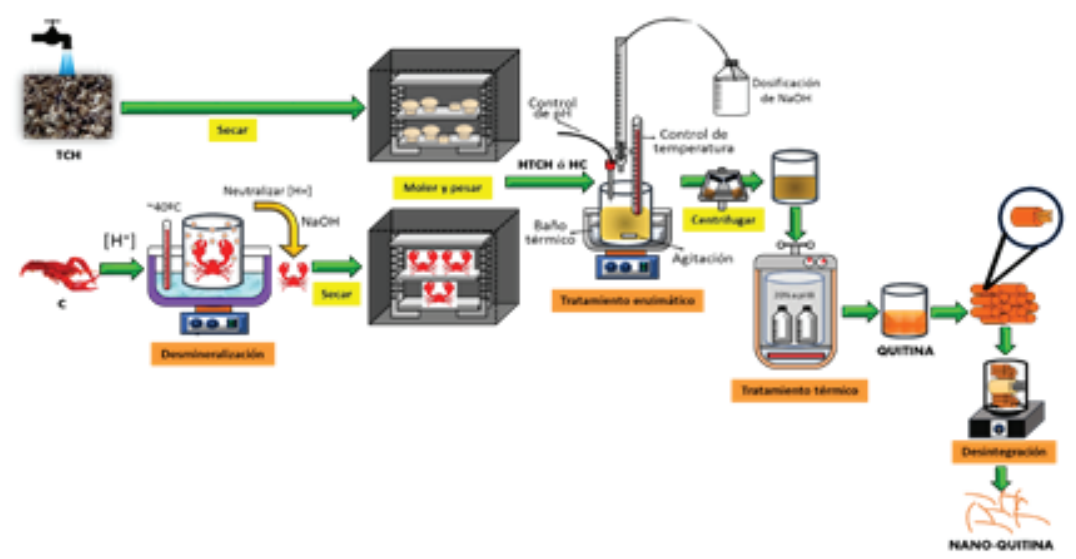

Figure 1

Esquema del procesamiento de quitina y nanoquitina.

este útilmo se utilizó un procedimiento previo para su desmineralización mediante un proceso fermentación 'in situ' del ácido láctico [13].

Se obtuvo fracciones de quitina de los dos subproductos siguiendo el procedimiento descrito en la Figura 1. La quitina cruda obtenida por estos procesos y una dilución 1:5 de hipoclorito en pasos sucesivos de $6 \mathrm{hr}$ a temperatura ambiente, dio una quitina de buena calidad, con una concentración del $83 \pm 1,8 \%$ y del $80 \pm 2,4 \%$ para $A$. bisporus y $P$. clarkii respectivamente, obteniéndose una quitina muy similar a una quitina estandar.

Estos resultados han sido comprobados mediante el análisis infrarrojo frente a una quitina comercial. Como se puede observar en la Figura 2 ( $a, b$ y c) los espectros infrarrojos muestran que la quitina obtenida mediante procesos biotecnológicos es muy similar a una quitina estándar.
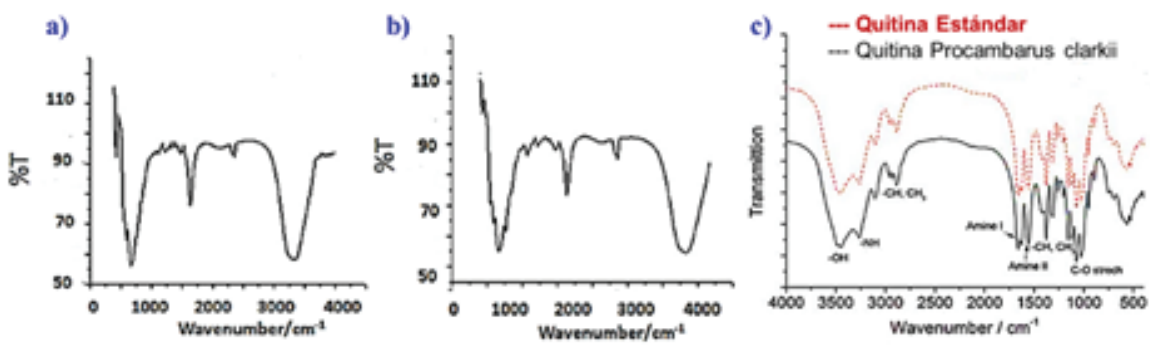

Figure 2

Espectros FTIR de quitina obtenidos de (a) Quitina estándar, (b) Quitina de Agaricus bisporus, (c) Quitina obtenida de Procambarus clarkii.

Durante el primer tratamiento en la preparación de la quitina se obtvo una serie de sustancias solubles como péptidos, oligopéptidos, aminoácidos, y en la parte insoluble quitina-glucanos en el caso de $A$. bisporus y quitina cruda con trazas de $\mathrm{CO}_{3} \mathrm{Ca}$ que no han sido eliminadas totalmente durante la desmineralización en el caso de $P$. clarkii. Al realizar el segundo tratamiento logramos extraer la quitina de la parte soluble y otros compuestos insolubles también de interés industrial. La separación del complejo quitina-glucano se lo realizó mediante digestión enzimática con una mezcla de $\alpha$-, 
$\beta$-glucanasas usando una relación $\mathrm{E} / \mathrm{S}$ de 0,02 , obteniéndose una parte soluble que contiene oligosacáridos, disacáridos y monosacáridos y la parte insoluble constituida por quitina solamente. Esta quitina al ser un polímero natural es biocompatible y biodegradable en el cuerpo, y puede ser utilizado para aplicaciones biomédicas y farmacéuticas, además puede ser utilizada como fuente de fermentación para la producción de quitinasas y/o como sustrato para la producción de quito-oligosacáridos con actividad inductora [5].

A partir de la quitina obtenida, y después de un ciclo de tratamiento con un molinillo de tipo húmedo se logró obtener fibras uniformes de quitina con un ancho de aproximadamente $8 \mathrm{~nm}$, manteniendo su estructura química y cristalina original [10, 18-20]. El desafío de este trabajo fue obtener nanofibras de quitina de pequeño diámetro y bajo contenido de proteínas. La longitud y el ancho de las nanofibras de acuerdo a la distribución estructural están relacionadas con la cantidad de proteína que se encuentra restante, es por esto que la disminución del contenido de proteínas permitió obtener una fracción mayor de fibrillas de quitina de menor diámetro y a su vez más cortas, siendo estas predominantes en las muestras. En la Figura 3 se muestra las nanofibras de quitina obtenidas.
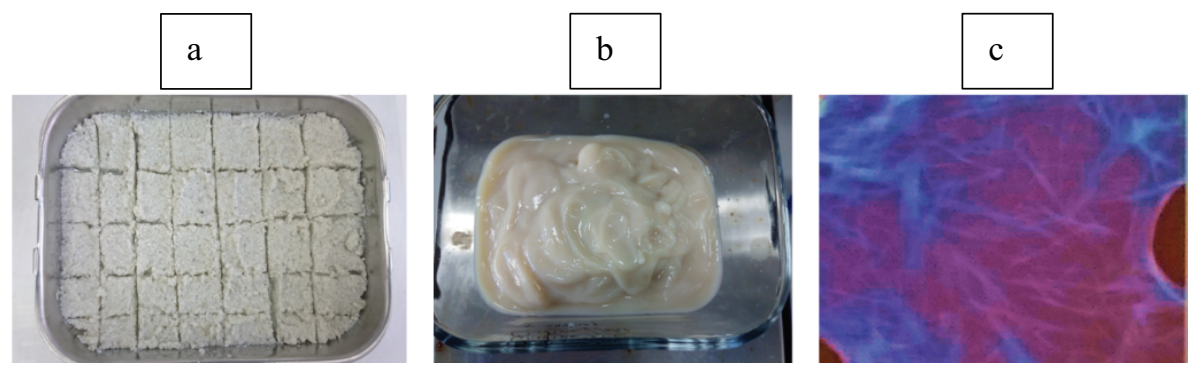

Figure 3

(a) Quitina, (b)Nanofibras de quitina, (c) Nanofibras de quitina observadas al microscopio.

En la Tabla 1 se muestran los resultados obtenidos de los tratamientos realizados para la extracción de proteínas, como se observa a medida que cambia el proceso y el tiempo, el porcentaje de proteínas extraídas es mayor, quedando en la muestra menor cantidad de proteína permitiendo una mayor fracción de nanofibras de quitina con un diámetro menor.

En la Figura 4 se puede observar que en las muestras donde mayor cantidad de proteínas se logró extraer, la transmitancia óptica es más alta, lo que indica que la transmitancia depende del diámetro de las nanofibras suspendidas [21]. Los mejores resultados se obtuvieron para DTeS2TT y TeS2TT.

\section{Discusión}

Como se ha mencionado anteriormente la extracción de quitina mediante tratamientos químicos tiene muchos inconvenientes principalmente el impacto sobre el medio ambiente debido a las sustancias químicas utilizadas durante el proceso, además que daña las propiedades fisicoquímicas de la quitina, es por ello que actualmente se 


\section{Table 1}

Cantidad de proteína extraída en cada proceso.

\begin{tabular}{ll|l} 
& Tratamiento & $\%$ Proteína extraído \\
PST & Procambarus clarkii Sin Tratamiento & $31,5 \pm 2,5^{\#}$ \\
DTe2 & Desmineralización + Tratamiento enzimático de $2 \mathrm{hr}$ & $40,5 \pm 0,8^{\#}$ \\
DTe4 & Desmineralización + Tratamiento enzimático de $4 \mathrm{hr}$ & $54,7 \pm 1,3^{\#}$ \\
DTeS4 & Desmineralización + Tratamiento enzimático secuencial de $4 \mathrm{hr}$ & $77,2 \pm 1,6^{\#}$ \\
DTeS2TT & Desmineralización + Tratamiento enzimático secuencial de $2 \mathrm{hr}+$ & $83,9 \pm 1,8^{\#}$ \\
& Tratamiento térmico & \\
AST & Agaricus bisporus Sin Tratamiento & $28,6 \pm 1,7^{\$}$ \\
Te2 & Tratamiento enzimático de $2 \mathrm{hr}$ & $41,3 \pm 1,6^{\$}$ \\
TeS4 & Tratamiento enzimático de $4 \mathrm{hr}$ & $49,4 \pm 2,0^{\$}$ \\
TeS2TT & Tratamiento enzimático secuencial de $4 \mathrm{hr}$ & $76,3 \pm 3,1^{\$}$ \\
\hline
\end{tabular}

${ }^{\$}$ Factor 5,45 para $A$. bisporus; ${ }^{*}$ Factor 6,24 para $P$. clarkii.

a)

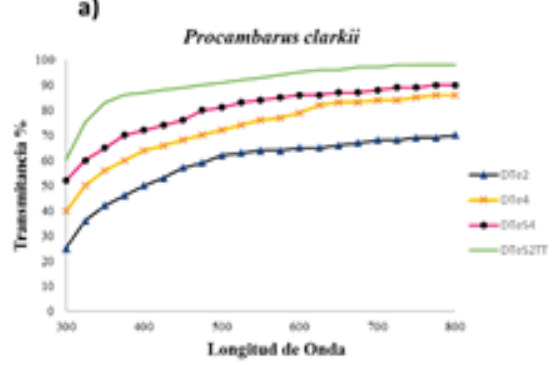

b)

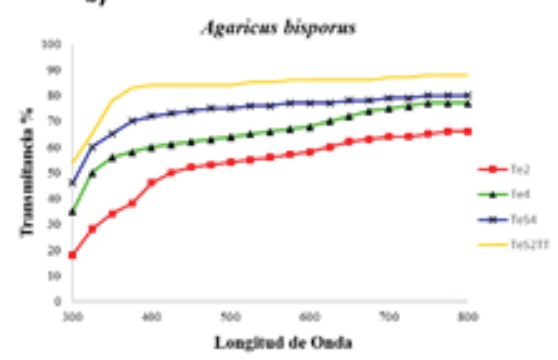

Figure 4

Transmitancia óptica de nanofibras de quitina, (a) Procambarus clarkii, (b) Agaricus bisporus.

está investigando la aplicación de enzimas y microorganismos para la extracción de quitina. Khanafari et al., estudiaron la extracción de quitina de los caparazones de camarones utilizando métodos químicos y biológicos, sus resultados indicaron que el método biológico (utilizando microorganismos) fue mejor que el químico porque conserva la estructura de la quitina [22]. Bustos y Healy también demostraron que la quitina obtenida de las cáscaras de camarones con diversos microorganismos proteolíticos tiene mayores pesos moleculares en comparación con la quitina de mariscos preparada químicamente. La mayoría de estudios recientes demuestran que los métodos biológicos presentan mayores ventajas sobre los métodos químicos, el método descrito en el presente estudio comprende un método biológico que ofrece una alta reproducibilidad, una manipulación más simple, un menor consumo de sustancias químicas y por lo tanto un menor aporte de energía, para la desproteinización se utilizó proteasas obtenidas de Bacillus licheniformis debido a que presenta algunas ventajas como disminuir los costos del proceso, preservar el medio ambiente y además que el hidrolizado que se obtiene es una buena fuente de aminoácidos esenciales en aplicaciones alimentarias. Teniendo en cuenta que este método no permite la extracción de una quitina netamente pura, es 
necesario un procedimiento adicional de purificación para ser aplicado en la industria médico-farmacéutica [23], debido a que pueden ser utilizados para la liberación de fármacos, en la ingeniería de tejidos y el vendaje para el cuidado de heridas, al ser un polímero natural, biocompatible, no tóxico, no alergénico y biodegradable en el cuerpo. Además, se usa como excipiente y vehículo de drogas en forma de película, gel o polvo para aplicaciones que involucran mucoadhesividad [24].

Los nanocristales de $\alpha$-quitina poseen un diámetro de $8-10 \mathrm{~nm}$, que corresponde al resultado obtenido en este estudio, se podría obtener un menor diámetro y a su vez una longitud más corta si durante la preparación de la quitina en lugar de realizar una hidrólisis simple se realizara una hidrólisis secuencial con el fin de extraer mayor cantidad de proteínas. El producto obtenido se puede utilizar para aplicaciones industriales en farmacia, cosmética, nuevos ingredientes de alimentos funcionales, agricultura, tratamientos de aguas residuales. Las nanofibras obtenidas podrían constituir como un medicamento nuevo o alimento funcional [25].

\section{Conclusiones}

Este estudio presenta un método favorable con el medio ambiente para la obtencion de quitina y nanoquitina. La concentración de quitina cruda obtenida, determinada como $\mathrm{N}$-acetil-glucosamina, fue de aproximadamente el $80 \%$ tanto de $A$. bisporus como de $P$. clarkii y muy similar a la disponible comercialmente. Se obtuvieron nanofibras de quitina uniformes con un ancho de aproximadamente $8 \mathrm{~nm}$, que aún mantienen sus estructuras químicas y cristalinas originales, la funcionalización de estas nanofibras permite su uso en diferentes materiales y compuestos, dentro de la industria farmacéutica, cosmética, agricultura y tratamientos de aguas residuales.

\section{Agradecimientos}

Agradecemos al Ministerio de Ciencia español e Innovación para el apoyo financiero de este trabajo (Proyecto RTC2015-4039-2), que cuenta con apoyo financiero parcial del FEDER fondos de la Unión Europea.

\section{Conflicto de Intereses}

Declaro que no existen intereses particulares por parte de los autores o de la entidad científica que pudiesen afectar directa o indirectamente a los resultados.

\section{References}

[1] Raabe D, Sachs C, Romano PJAM. The crustacean exoskeleton as an example of a structurally and mechanically graded biological nanocomposite material. Acta Materialia 2005;53(15):4281-4292.

[2] García Mendoza C, Sanchez E, Novaes-Ledieu M. Differences in microfibrils in the walls of Agaricus bisporus secondary mycelium. FEMS Microbiology Letters 1987;44:161-165. 
[3] Ifuku S, Nomura R, Morimoto M, Saimoto H. Preparation of chitin nanofibers from mushrooms. Materials 2011;4(8):1417-1425.

[4] Muzzarelli RA, El Mehtedi M, Mattioli-Belmonte M. Emerging biomedical applications of nano-chitins and nano-chitosans obtained via advanced eco-friendly technologies from marine resources. Marine Drugs 2014;12(11):5468-5502.

[5] Urbina-Salazar A, Inca-Torres AR, Falcón-García G, et al. Chitinase production by Trichoderma harzianum grown on a chitin-rich mushroom byproduct formulated medium. Waste and Biomass Valorization 2018;10(10):2915-2923.

[6] Urbina-Salazar A. Producción de quitinasas a partir de subproductos de la industria alimentaria: Aplicación a los Hongos comestibles y Crustáceos [Tesis Doctoral]. Sevilla, España: Universidad de Sevilla; 2019.

[7] Lopes C, Antelo L, Franco-Uría A, Alonso A, Perez-Martín R. Chitin production from crustacean biomass: Sustainability assessment of chemical and enzymatic processes. Journal of Cleaner Production 2017;112.

[8] Salaberria AM, Diaz RH, Labidi J, Fernandes SC. Role of chitin nanocrystals and nanofibers on physical, mechanical and functional properties in thermoplastic starch films. Food Hydrocolloids 2015;46:93102.

[9] Xia Y, Yang P, Sun Y, et al. One-dimensional nanostructures: Synthesis, characterization, and applications. Advanced Materials 2003;15(5):353-389.

[10] Li D, Xia Y. Electrospinning of nanofibers: Reinventing the wheel? Advanced Materials 2004;16(14):11511170 .

[11] Lopez O, Garcia MA, Villar MA, Gentili A, Rodriguez MS, Albertengo L. Thermo-compression of biodegradable thermoplastic corn starch films containing chitin and chitosan. LWT-Food Science and Technology 2014;57(1):106-115.

[12] Gopalan Nair K, Dufresne A. Crab shell chitin whisker reinforced natural rubber nanocomposites. 1. Processing and swelling behavior. Biomacromolecules 2003;4(3):657-665.

[13] Bautista J, Jover M, Gutierrez JF, et al. Preparation of crayfish chitin by in situ lactic acid production. Process Biochemistry 2001;37(3):229-234.

[14] Inca-Torres AR, Urbina-Salazar AR, Falcón-García G, et al. Hydrolytic enzymes production by Bacillus licheniformis growth on fermentation media formulated with sewage sludge. Journal of Biotech Research 2018;9:14-26.

[15] Carroad P, Tom R. Bioconversion of shellfish chitin wastes: Process. Conception and Selection of Microorganisms. Journal of Food Science 1978;43:1158-1161.

[16] Dubois M, Gilles KA, Hamilton JK, Rebers PT, Smith F. Colorimetric method for determination of sugars and related substances. Analytical Chemistry 1956;28(3):350-356.

[17] AOAC. Official methods of analysis. 13th ed. Washington, DC: Association of Official Analytical Chemists; 1995.

[18] Michalenko GO, Hohl HR, Rast D. Chemistry and architecture of the mycelial wall of Agaricus bisporus. Microbiology 1976;92(2):251-262.

[19] Zivanovic S, Buescher R, Kim SK. Mushroom texture, cell wall composition, color, and ultrastructure as affected by $\mathrm{pH}$ and temperature. Journal of Food Science 2003;68(5):1860-1865.

[20] Ivshina TN, Artamonova SD, Ivshin VP, Sharnina FF. Isolation of the chitin-glucan complex from the fruiting bodies of mycothallus. Applied Biochemistry and Microbiology 2009;45(3):313-318.

[21] Fukuzumi H, Saito T, Iwata T, Kumamoto Y, Isogai A. Transparent and high gas barrier films of cellulose nanofibers prepared by TEMPO-mediated oxidation. Biomacromolecules 2009;10(1):162-165.

[22] Khanafari A, Marandi Reza, Sanati S. Recovery of chitin and chitosan from shrimp waste by chemical and microbial methods. Iranian Journal of Environmental Health Science \& Engineering 2008;5:19-24.

[23] Younes I, Rinaudo M. Chitin and chitosan preparation from marine sources. Structure, properties and applications. Marine Drugs 2015;13(3):1133-1174.

[24] Gortari MC, Hours RA. Biotechnological processes for chitin recovery out of crustacean waste: a minireview. Electronic Journal of Biotechnology 2013;16(3):14-14.

[25] Azuma K, Osaki T, Wakuda T, et al. Beneficial and preventive effect of chitin nanofibrils in a dextran sulfate sodium-induced acute ulcerative colitis model. Carbohydrate Polymers 2012;87(2):1399-1403. 